\title{
Interactions between Cytosolic Phospholipase A2 Activation and Mitochondrial Reactive Oxygen Species Production in the Development of Ventilator-Induced Diaphragm Dysfunction
}

\author{
Xian-Long Zhou, ${ }_{1}$ Xiao-Jun Wei, ${ }^{1}$ Shao-Ping Li, ${ }^{1}$ Rui-Ning Liu, ${ }^{1}$ Ming-Xia Yu $\mathbb{D}^{2}{ }^{2}$ \\ and Yan Zhao ${ }^{1}$ \\ ${ }^{1}$ Emergency Center, Zhongnan Hospital of Wuhan University, 169 Donghu Road, Wuhan, Hubei 430071, China \\ ${ }^{2}$ Department of Clinical Laboratory, Zhongnan Hospital of Wuhan University, 169 Donghu Road, Wuhan, Hubei 430071, China \\ Correspondence should be addressed to Yan Zhao; doctoryanzhao@whu.edu.cn
}

Received 3 January 2019; Accepted 13 March 2019; Published 18 April 2019

Academic Editor: Demetrios Kouretas

Copyright @ 2019 Xian-Long Zhou et al. This is an open access article distributed under the Creative Commons Attribution License, which permits unrestricted use, distribution, and reproduction in any medium, provided the original work is properly cited.

Cytosolic phospholipase A2 (cPLA2) has been reported to be critical for infection-induced mitochondrial reactive oxygen species (ROS) production and diaphragm dysfunction (DD). In the present study, we aim to investigate whether cPLA2 was involved in ventilator-induced diaphragm dysfunction (VIDD). Our results showed that mechanical ventilation (MV) induced cPLA2 activation in the diaphragm with excessive mitochondrial ROS generation and muscle weakness. Specific inhibition of cPLA2 with CDIBA resulted in decreased mitochondrial ROS levels and improved diaphragm forces. In addition, mitochondriatargeted antioxidant MitoTEMPO attenuated ventilator-induced mitochondrial oxidative stress and downregulated cPLA2 activation in vivo. Both CDIBA and MitoTEMPO were able to attenuate protein degradation, muscle atrophy, and weakness following prolonged MV. Furthermore, laser Doppler imaging showed that MV decreased diaphragm tissue perfusion and induced subsequent hypoxia. An in vitro study also demonstrated a positive association between cPLA2 activation and mitochondrial ROS generation in $\mathrm{C} 2 \mathrm{C} 12$ cells cultured under hypoxic condition. Collectively, our study showed that cPLA2 activation positively interacts with mitochondrial ROS generation in the development of VIDD, and ventilator-induced diaphragm hypoxia serves as a possible contributor to this positive feedback loop.

\section{Introduction}

Diaphragm dysfunction (DD) occurring following long-term mechanical ventilation (MV), which is widely known as ventilator-induced mechanical ventilation (VIDD), is a great concern in the modern era of MV. Several studies showed that controlled MV (CMV) leads to decreased diaphragmatic force-generating capacity in various animal models [1, 2]. In vitro measurements of diaphragm contractile properties suggest that the decrease in contractility is an early and progressive phenomenon [3]. Physiologically, CMV induces diaphragm atrophy as early as 12 hours after induction of MV, and the atrophy develops rapidly [4]. Numerous evidences suggested that, in addition to protein synthesis, proteolysis plays a dominant role in diaphragm atrophy after CMV [5]. In addition, the onset of oxidative injury is rapid and long-lasting in animal MV models and has been demonstrated to be a key upstream regulator of protease system activation that contributes to diaphragm muscle atrophy and weakness [6].

Currently, it has been commonly accepted that mitochondria are the major source of reactive oxygen species (ROS) generation that induces the activation of protease and subsequent diaphragm atrophy during MV [7]. A recent study also suggested that diaphragm mitochondrial ROS formation, which mediated diaphragm weakness, may be critically dependent on PLA2 activation [8]. In addition, cPLA2 has been found to modulate cytokine-induced calpain activation in $\mathrm{C} 2 \mathrm{C} 12$ cells and infection-induced diaphragm weakness in animals [9]. These results indicate that cPLA2 activation possibly induces acute oxidant injury and increases calpain activity, 
which finally results in DD. Moreover, a previous study showed that prolonged MV results in a time-dependent decrease in the ability of the diaphragm to augment blood flow to match $\mathrm{O}_{2}$ demand in response to contractile activity [10]. This study was attempting to speculate that the ventilator-induced decrease in diaphragmatic oxygenation could promote a hypoxia-induced generation of ROS in diaphragm muscle fibers and contribute to VIDD. However, whether CPLA2 activation contributes to oxidative stress and the development of VIDD has not yet been reported. In the present study, we aim to investigate whether cPLA2 activation contributes to ventilator-induced mitochondrial ROS generation and DD and to investigate the mechanism by which the ventilator induces CPLA2 activation.

\section{Methods and Materials}

2.1. Animals, Cell Line, and Reagents. Adult male Wistar rats (SPF level), weighing 450 to $550 \mathrm{~g}$, were purchased from the Charles River Laboratories (Beijing, China). All animal studies were performed in the Bio-Safety Level III Laboratory of Wuhan University (Wuhan, Hubei, China).

All animals were kept in cages under controlled conditions (temperature: $25^{\circ} \mathrm{C} \pm 2^{\circ} \mathrm{C}$; relative humidity: $50 \% \pm 5 \%$ ) with a $12: 12$ light-dark cycle. Water and food were provided to the animals ad libitum. Animal experiments were performed in accordance with the Guidelines of Animal Care and Use. This study was approved by the Animal Experiment Center of Zhongnan Hospital of Wuhan University.

The $\mathrm{C} 2 \mathrm{C} 12$ cell line was purchased from Procell (\#CL0044, Wuhan, Hubei, China). C2C12 cells were cultured in Dulbecco's modified Eagle medium (DMEM; Invitrogen, Carlsbad, CA) supplemented with $10 \%$ fetal bovine serum (FBS) and penicillin/streptomycin at $37^{\circ} \mathrm{C}$ and $5 \% \mathrm{CO}_{2}$ in a humidified chamber.

Pentobarbital sodium was purchased from AMRESCO (Cleveland, OH, USA). Krebs-Henseleit bicarbonate buffer was purchased from M\&C Gene Technology Ltd. (Beijing, China). The cPLA2 activity assay kit was purchased from Cayman Chemical (765021-96T, MI, USA). A MitoSOX Red mitochondrial superoxide indicator was purchased from Yaesen (\#40778ES50, Shanghai, China). A Hydrogen Peroxide Colorimetric/Fluorometric Assay Kit was purchased from BioVision (Palo Alto, CA, USA). AACOCF3 was purchased from APEXBIO (B6748, Shanghai, China). SS-31 was purchased from Chinapeptides (Shanghai, China). MitoTEMPO was purchased from Enzo Life Sciences (NY, USA). CDIBA was purchased from Axon Medchem (VA, USA). Primary antibodies including anti-cPLA2, anti-pcPLA2, anti-Atrogin-1, anti-MuRF1, anti-HIF- $1 \alpha$, anti-Slow Skeletal Myosin Heavy Chain (NOQ7.5.4D), anti-Fast Myosin Skeletal Heavy Chain (MY-32), and anti-Laminin were purchased from Abcam (Shanghai, China).

\subsection{Study Protocol}

2.2.1. Animal Study. To investigate whether prolonged MV induces CPLA2 activation in the diaphragm and the roles of cPLA2 activation in ventilator-induced ROS generation and diaphragm muscle weakness, animals were assigned to the following groups: (1) control group $(n=5)$ : animals received sham operation without ventilation; (2) mechanical ventilation group (PMV, $n=5$ ): animals received MV for 12 hours; (3) MV+CDIBA group $(n=5)$ : animals received MV for 12 hours with a single injection of CDIBA (a cPLA2 inhibitor, intraperitoneally (IP), $30 \mathrm{mg} / \mathrm{kg}^{-1}$ body weight) at the onset of ventilation; then, a continuous intravenous injection of CDIBA $\left(5 \mathrm{mg} / \mathrm{kg}^{-1} / \mathrm{h}^{-1}\right)$ was given throughout this study; (4) $\mathrm{MV}+\mathrm{MitoT}$ group $(n=5)$ : animals received MV for 12 hours with a single injection of MitoTEMPO (a mitochondriatargeted antioxidant, IP, $30 \mathrm{mg} / \mathrm{kg}^{-1}$ ) at the onset of ventilation; then, an intravenous injection of MitoTEMPO $\left(10 \mathrm{mg} / \mathrm{kg}^{-1} / \mathrm{h}^{-1}\right)$ was given during experiment; and (5) MV + CDIBA+MitoT group $(n=5)$ : animals received MV for 12 hours with both CDIBA and MitoTEMPO treatments.

2.2.2. Cell Study. Cells were seeded into four-well rectangular plates, the surface of which was coated with Matrigel (Becton, Dickinson and Co., Franklin Lakes, NJ, USA), at a density of $2.5 \times 10^{5}$ cells/well, with $3 \mathrm{~mL}$ of DMEM $(25 \mathrm{mM}$ glucose; Invitrogen, Carlsbad, CA, USA) supplemented with $10 \%$ FBS and $1 \%$ penicillin-streptomycin. The cells were maintained in an incubator at $37^{\circ} \mathrm{C}$ under a $5 \% \mathrm{CO}_{2}$ atmosphere. Then, cells were cultured under hypoxia for $0,2,4,8$, and 12 hours to create an ex vivo hypoxia model. In a parallel study, C2C12 cells cultured under hypoxia for 12 hours with cPLA2-specific inhibitor AACOCF3 $(15 \mu \mathrm{M})$ or mitochondria-targeted antioxidant SS31 $(10 \mu \mathrm{g} / \mathrm{mL})$. At the end of hypoxia, western blot assay was performed for the measurements of p-cPLA2, cPLA2, and HIF- $1 \alpha$. Cell mitochondrial ROS levels were analyzed using a MitoSOX Red mitochondrial superoxide indicator. The CPLA2 and calpain activities were also measured.

2.3. In Vitro Hypoxia Model. A cell hypoxia model was established using a modular incubator chamber as previously reported [11]. In brief, cell cultures are placed in the hypoxic chamber. At the same time, a Petri dish containing sterile water has also been placed in the chamber to provide adequate humidification. The "twin" cell culture in normoxia is prepared as a control. Then, the chamber is closed to create hypoxia, and tubing is attached to a "hypoxia tank" containing a $1 \% \mathrm{O}_{2}$ gas mixture. Finally, the chamber is returned to a conventional incubator for the desired period of time.

2.4. Mechanical Ventilation. An animal mechanical ventilation model was established and modified as previously described [10]. In detail, animals were anesthetized with sodium pentobarbital $(40 \mathrm{mg} / \mathrm{kg}$ body weight, IP). After anesthesia, animals were placed on a recirculating heating blanket and fixed. Then, animals were tracheostomized and connected to a volume-driven small animal ventilator (VentElite, Harvard Apparatus; Cambridge, MA, USA). The tidal volume (TV) was set at $5 \mathrm{~mL} / \mathrm{kg}$ body weight, and the respiratory rate (RR) was set at 55 to 60 breaths/min. Breathing air was humidified and enriched with oxygen. To avoid ventilator-induced systemic hypoxia, the ventilator parameters and oxygen flow were adjusted to maintain $\mathrm{PaO}_{2}$ 
between 80 and $100 \mathrm{mmHg}$ and $\mathrm{PaCO}_{2}$ between 35 and $45 \mathrm{mmHg}$ during the entire study. The values of $\mathrm{PaO}_{2}$ and $\mathrm{PaCO}_{2}$ were determined through arterial blood gas analysis, which was performed every 2 hours during the study. Blood pressure (BP) and heart rate (HR) were monitored at the tail using tail cuff plethysmography (BP-2010 Series Blood Pressure Meter, Softron, Japan). The right jugular vein was cannulated for continuous infusion of normal saline (Baxter, Deerfield, IL) at a rate of $1 \mathrm{~mL} / \mathrm{kg}^{-1} / \mathrm{h}^{-1}$ and pentobarbital sodium $\left(10 \mathrm{mg} / \mathrm{kg}^{-1} / \mathrm{h}^{-1}\right)$ using an electric pump. Body temperature was maintained at $37^{\circ} \mathrm{C}$ during the experiment by external warming with a homeothermic blanket system.

2.5. Laser Doppler. Diaphragm muscle blood flow was measured using laser Doppler (PeriFlux System 5000). The diaphragm was exposed by midline laparotomy, and the probe of the laser Doppler was applied perpendicularly against the dorsal part of the diaphragm. The soleus was exposed after careful dissection of the right leg. The probe was then applied perpendicularly against the soleus muscle. Diaphragmatic signal analysis was restricted to the end-expiratory phase to avoid any influence of diaphragm motion on the recordings. The information is picked up by the returning fiber and displayed as perfusion units (PU).

2.6. Blood Sample Analysis. Arterial blood samples were collected every 2 hours for blood gas analysis during mechanical ventilation. Ventilator settings were adjusted according to blood gas results to maintain appropriate $\mathrm{PaO}_{2}$ and $\mathrm{PaCO}_{2}$. Blood gas analysis was performed using an i-STAT1 Analyzer (Abbott, Kyoto, Japan). Blood cell counts were performed using an automatic blood cell analyzer Pentra MS CRP (HORIBA Medical, Kyoto, Japan).

2.7. Cytosolic Phospholipase A2 Activity Assay. cPLA2 activities were determined as previously described [8]. In detail, tissue and cell samples were prepared by homogenization in buffer and subsequent protein determination of homogenates. Samples, blanks, and a positive control were loaded on a 96-well plate. Then, substrates were added into each well and incubated in the dark for 1 hour. The DTNB mixture $(10 \mu \mathrm{L})$ was added and well mixed. After 5 minutes, optical density was read at $414 \mathrm{~nm}$ and activity was calculated.

2.8. Calpain Activity Assay. Calpain activities were determined using a commercial kit (Abcam) according to the manufacturer's instructions. In brief, tissue and cell samples were prepared by homogenization in buffer and subsequent protein determination of homogenates. Then, samples, and positive and negative controls, were added into a 96-well plate. Then, the $10 \mathrm{x}$ reaction buffer and calpain substrate were added into each well and incubated in the dark for 30 minutes at $37^{\circ} \mathrm{C}$. Fluorescence at Ex $400 \mathrm{~nm} / \mathrm{Em} 505 \mathrm{~nm}$ was measured.

2.9. Measurements of Muscle Contractile Properties. Each muscle strip was rapidly mounted in a tissue chamber containing Krebs-Henseleit $(\mathrm{K}-\mathrm{H})$ solution. The solution was bubbled with a gas mixture of $95 \% \quad \mathrm{O}_{2}-5 \% \quad \mathrm{CO}_{2}$ and maintained at $27^{\circ} \mathrm{C}$ and $\mathrm{pH}$ 7.4. Muscle extremities were held in spring clips and attached to an electromagnetic force transducer. Diaphragm muscle strips were electrically stimulated in twitch and tetanus with two silver electrodes positioned parallel to the muscle and delivering electrical stimulation lasting $1 \mathrm{~ms}$. Muscle strips recovered their optimal mechanical performance after a 20-minute equilibration period. Strips were stimulated in twitch and then sequentially in tetanus with trains of $10,20,40,60,80$, 100 , and $120 \mathrm{~Hz}$ stimuli, and force and shortening were recorded. Fatigability was assessed by means of $330 \mathrm{~ms}$ stimulations repeated at $25 \mathrm{~Hz}$ and applied every second over a 5-minute period. Forces were measured at 2 minutes after stimulation, and the fatigue index (FI) was calculated as force at the onset of stimulation $\left(F_{0}\right) /$ force at 2 minutes after stimulation $\left(F_{2 \mathrm{~min}}\right)$. At the end of the experiment, each muscle cross-sectional area (in $\mathrm{cm}^{2}$ ) was calculated from the ratio of muscle weight to muscle length at $L_{\max }$, assuming a muscle density of 1.06. All analyses were performed from digital records of force and length obtained with a computer.

2.10. Detection of Cellular Mitochondrial ROS Production. Mitochondrial ROS levels in cells were measured using a MitoSOX Red mitochondrial superoxide indicator. DMSO $13 \mu \mathrm{L}$ was added into $50 \mu \mathrm{g}$ of MitoSOX Red mitochondrial superoxide indicator and mixed well. MitoSOX Red mitochondrial superoxide indicator $(5 \mathrm{mM})$ was diluted with Hank's balanced salt solution (HBSS) buffer into a final concentration of $5 \mu \mathrm{M}$ (working solution). Cells were soaked on a cover glass with 1 to $2 \mathrm{~mL}$ of working solution and cultured for 10 minutes under $37^{\circ} \mathrm{C}$. Cells were washed with prepared buffers three times. Staining and observation were conducted under a microscope.

2.11. Detection of Diaphragm Mitochondrial $\mathrm{H}_{2} \mathrm{O}_{2}$ Generation. Mitochondrial isolation was performed as previously described [12]. Then, mitochondrial $\mathrm{H}_{2} \mathrm{O}_{2}$ levels were detected using a commercial Hydrogen Peroxide Assay Kit (BioVision Inc., CA, USA) according to the manufacturer's instruction. The absorbance was determined at $570 \mathrm{~nm}$.

2.12. Western Blotting. Proteins were extracted from tissue samples using a protein extraction reagent (Sigma, USA), following a protocol provided by the manufacturer. Cells were lysed in radioimmunoprecipitation assay (RIPA; P0013B, Beyotime Biotechnology, Shanghai, China), and protein concentrations were determined using the BCA protein assay kit (Beyotime, Shanghai, China). Equal amounts of proteins were resolved by sodium dodecyl sulfate polyacrylamide gel electrophoresis (SDS-PAGE), and the proteins were transferred to Hybond ECL membranes (Amersham, Buckinghamshire, UK). The membranes were incubated with primary antibodies including anti-cPLA2, anti-p-cPLA2, antiAtrogin-1, anti-MuRF-1, and anti-HIF- $1 \alpha$ at $4^{\circ} \mathrm{C}$ overnight. After washing with TBST, the membranes were probed with HRP-labeled secondary antibodies. The membranes were visualized using an enhanced chemiluminescence system (Kodak, Rochester, NY, USA). Glyceraldehyde-3-phosphate dehydrogenase (GAPDH) was used as a loading control. 
TABLE 1: Arterial blood gas analysis at the end of study (mean \pm SD).

\begin{tabular}{lccccc}
\hline & Control $(n=5)$ & MV $(n=5)$ & MV+CDIBA $(n=5)$ & MV+MitoT $(n=5)$ & MV+CDIBA+MitoT $(n=5)$ \\
\hline $\mathrm{pH}$ & $7.40 \pm 0.01$ & $7.38 \pm 0.03$ & $7.39 \pm 0.05$ & $7.40 \pm 0.05$ & $7.38 \pm 0.02$ \\
$\mathrm{PaCO}_{2}(\mathrm{mmHg})$ & $42 \pm 5$ & $41 \pm 5$ & $39 \pm 4$ & $41 \pm 6$ & $39 \pm 5$ \\
$\mathrm{PaO}_{2}(\mathrm{mmHg})$ & $101 \pm 11$ & $98 \pm 10$ & $101 \pm 16$ & $102 \pm 8$ & $94 \pm 12$ \\
$\mathrm{HCO}_{3}{ }^{-}(\mathrm{mM})$ & $24.2 \pm 4.1$ & $23.9 \pm 3.2$ & $23.0 \pm 4.3$ & $22.6 \pm 3.8$ & $17.9 \pm 4.7$ \\
$\mathrm{BE}$ & $2.9 \pm 2.0$ & $2.1 \pm 1.3$ & $3.0 \pm 2.3$ & $2.5 \pm 1.1$ & $2.9 \pm 2.4$ \\
Lactate $(\mathrm{mM})$ & $1.25 \pm 0.22$ & $1.87 \pm 0.67$ & $1.85 \pm 0.24$ & $1.65 \pm 0.40$ & $1.80 \pm 0.69$ \\
\hline
\end{tabular}

$\mathrm{MV}=$ mechanical ventilation; CDIBA = 4-\{2-[5-chloro-1-(diphenylmethyl)-2-methyl-1H-indol-3-yl]-ethoxy $\}$ benzoic acid (cPLA2 inhibitor), a cPLA2 specific inhibitor; MitoT $=$ MitoTEMPO, a mitochondria-targeted antioxidant; $\mathrm{BE}=$ base excess.

2.13. Immunofluorescence Staining. In the present study, immunofluorescence staining was performed in a blinded manner on paraffin-embedded tissues to evaluate the crosssectional area of muscle fibers as previously described [13]. NOQ7.5.4D antibody was used for the identification of the slow myosin heavy chain, and the MY-32 antibody was used for the fast myosin heavy chains.

2.14. Statistical Analysis. Data are expressed as mean \pm standard deviation (SD). Between-group comparisons were performed with one-way or two-way repeated measures analysis of variance followed, when significant, by a post hoc Tukey test for normally distributed data (KolmogorovSmirnov test) and a Kruskal-Wallis test followed by the Dunn post hoc test for nonnormally distributed data [14]. All statistical analyses were performed using GraphPad 5 (GraphPad Software, La Jolla, CA). All $p$ values were twotailed, and a $p$ value less than 0.05 was considered significant.

\section{Results}

3.1. Systemic Response of Animals to $M V$. Arterial blood pressure, heart rate, and body temperature were monitored throughout the experiment, and no significant differences were observed between ventilation groups at the end of study $(p>0.05$, respectively) (sTable 1$)$. Blood gases and lactate levels are summarized in Table 1. Differences in $\mathrm{pH}$ values, bicarbonate, $\mathrm{PaO}_{2}$, and $\mathrm{PaCO}_{2}$ levels were insignificant between groups. In addition, there was no significant difference in lactate levels between all experimental groups $(p>0.05$, respectively). As shown in Table 2, blood cell counts had slight increases in leukocytes and neutrophils in the MV, MV+CDIBA, MV+MitoT, and MV+CDIBA + MitoT groups compared with controls. However, the differences were insignificant $(p>0.05$, respectively). Together, MV did not induce acid-base imbalance and systemic infections in animals in the present study.

3.2. Positive Interactions between cPLA2 Activation and Mitochondrial ROS Production in Diaphragm following MV. Here, we aim to determine the effects of cPLA2 in MVinduced mitochondrial ROS generation in the diaphragm. As seen in Figures 1(a) and 1(b), cPLA2-specific inhibitor CDIBA significantly reduced CPLA2 activation in the rat diaphragm following 12 hours of MV. Interestingly, administration of mitochondria-targeted antioxidant MitoTEMPO also downregulated ventilator-induced cPLA2 activation in the diaphragm $(p<0.05$, respectively). To investigate the effects of cPLA2 activation in mitochondrial ROS generation, we measured mitochondrial $\mathrm{H}_{2} \mathrm{O}_{2}$ production in the present study. Our results showed that mitochondrial $\mathrm{H}_{2} \mathrm{O}_{2}$ production in the diaphragm was significantly increased after 12 hours of MV. Importantly, mitochondrial $\mathrm{H}_{2} \mathrm{O}_{2}$ production was downregulated by the inhibition of cPLA2 activation with CDIBA (Figure 1(c)) $(p<0.05)$. Correlation analysis demonstrated a positive association between the cPLA2 activity and mitochondrial $\mathrm{H}_{2} \mathrm{O}_{2}$ generation levels $(r=0.974, p<0.0001)$ (Figure $1(\mathrm{~d}))$. Therefore, these results demonstrated a positive association between cPLA2 activation and mitochondrial ROS generation.

\subsection{Inhibition of cPLA2 Activation and Mitochondria-} Targeted Antioxidant Attenuated Ventilator-Induced Protein Degradation, Muscle Atrophy, and Weakness. Since mitochondrial ROS generation is the key regulator of proteolysis in the diaphragm following MV, proteolytic markers were determined for the evaluation of protein degradation. As seen in Figure 2(a), calpain activities were significantly increased in the MV group compared with controls. Specific inhibition of CPLA2 activation with CDIBA or mitochondria-targeted antioxidant MitoTEMPO significantly downregulated calpain activities in the diaphragm $(p<0.05$, respectively). The combination of CDIBA and MitoTEMPO further decreased diaphragm calpain activities. Moreover, western blot analysis demonstrated similar changes in atrophy-related genes Atrogin-1 and MuRF-1 as that of calpain in the diaphragm following $\mathrm{MV}$ (Figure 2(b)). Immunofluorescence staining suggested that MV induced apparent muscle atrophy, as the CSA of diaphragm muscle fibers were significantly decreased after 12 hours of $\mathrm{MV}$ in all ventilated animals, whereas CDIBA and/or MitoTEMPO treatments increased CSA of muscle fibers (Figures 2(c) and 2(e)). Moreover, in vitro measurements of muscle strip contractile properties demonstrated significant decreases of muscle forces in animals that underwent MV. However, administration of either CDIBA or MitoTEMPO apparently attenuated muscle weakness with increased maximal tetanic forces, force-frequency curves, and fatigue tolerances compared with the MV group (Figure 3). Collectively, these data confirmed that cPLA2 
TABLE 2: Blood cell counts at the end of the study (mean \pm SD).

\begin{tabular}{lccccc}
\hline & Control $(n=5)$ & MV $(n=5)$ & MV+CDIBA $(n=5)$ & MV+MitoT $(n=5)$ & MV+CDIBA+MitoT $(n=5)$ \\
\hline Erythrocytes $\left(\times 10^{12} / \mathrm{L}\right)$ & $5.64 \pm 0.21$ & $5.41 \pm 0.33$ & $5.39 \pm 0.15$ & $5.40 \pm 0.05$ & $5.30 \pm 0.26$ \\
Hemoglobin $(\mathrm{g} / \mathrm{L})$ & $132 \pm 21$ & $129 \pm 25$ & $139 \pm 34$ & $131 \pm 26$ & $129 \pm 15$ \\
Leukocytes $\left(\times 10^{9} / \mathrm{L}\right)$ & $6.72 \pm 0.52$ & $6.95 \pm 1.21$ & $6.81 \pm 0.67$ & $6.92 \pm 1.11$ & $7.04 \pm 0.57$ \\
Neutrophils $\left(\times 10^{9} / \mathrm{L}\right)$ & $3.21 \pm 0.12$ & $3.28 \pm 0.89$ & $3.30 \pm 0.63$ & $3.26 \pm 0.18$ & $3.29 \pm 0.07$ \\
Platelets $\left(\times 10^{9} / \mathrm{L}\right)$ & $112 \pm 12$ & $119 \pm 21$ & $111 \pm 11$ & $118 \pm 22$ & $109 \pm 12$
\end{tabular}

MV = mechanical ventilation; CDIBA = 4-\{2-[5-chloro-1-(diphenylmethyl)-2-methyl-1H-indol-3-yl]-ethoxy $\}$ benzoic acid (cPLA2 inhibitor), a cPLA2 specific inhibitor; MitoT = MitoTEMPO, a mitochondria-targeted antioxidant.

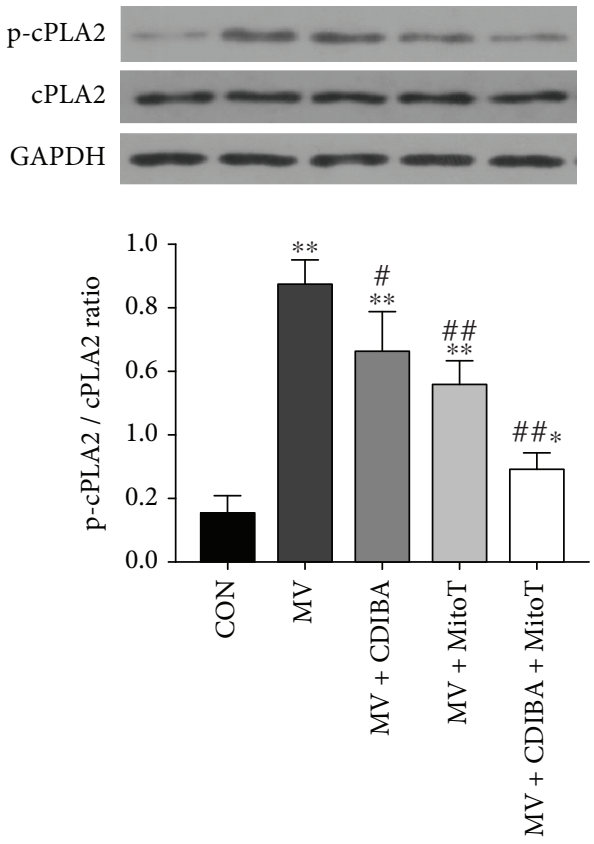

(a)

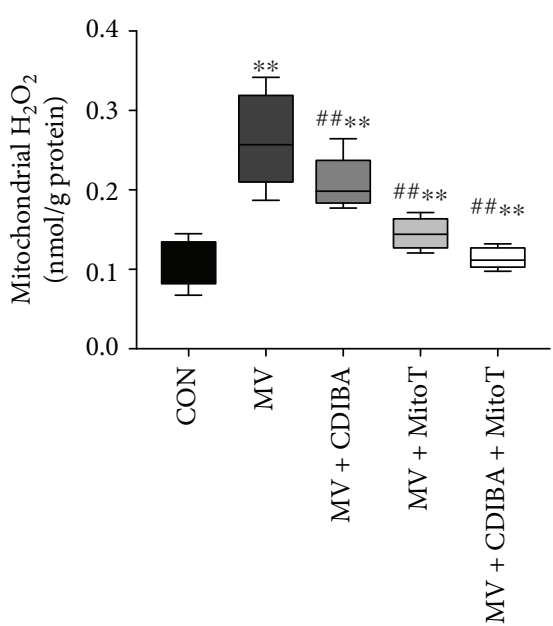

(c)

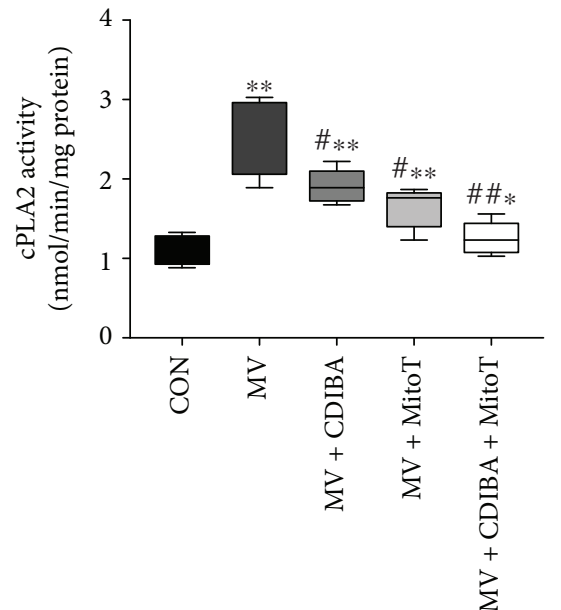

(b)

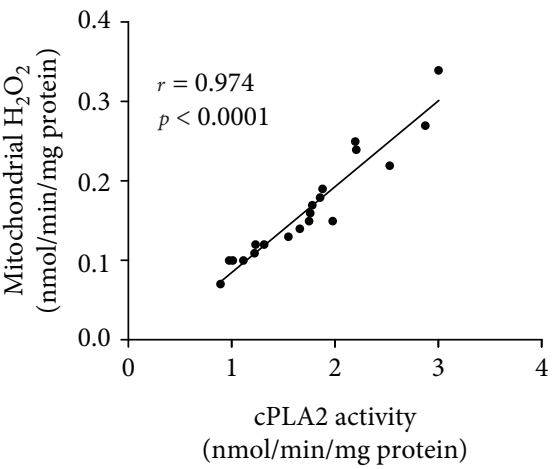

(d)

FIGURE 1: Interactions between cPLA2 and mitochondrial ROS generation in the diaphragm following MV. (a) Western blots for cPLA2 and p-cPLA2 in the diaphragm; (b) cPLA2 activity assay; (c) mitochondrial $\mathrm{H}_{2} \mathrm{O}_{2}$ generation in the diaphragm; (d) correlation analysis between cPLA2 activities and mitochondrial $\mathrm{H}_{2} \mathrm{O}_{2}$ levels. $\mathrm{MV}=$ mechanical ventilation; cPLA2 = cytosolic phospholipase $\mathrm{A} 2$; $\mathrm{CDIBA}=4$ - $\{2-[5-$ chloro-1-(diphenyl-methyl)-2-methyl-1H-indol-3-yl]-ethoxy benzoic acid, a specific cPLA2 inhibitor; MitoTEMPO=a mitochondriatargeted antioxidant. ${ }^{*} p<0.05$ and ${ }^{* *} p<0.01$ vs. the control group; ${ }^{\#} p<0.05$ and ${ }^{\# \#} p<0.01$ vs. the MV group. 


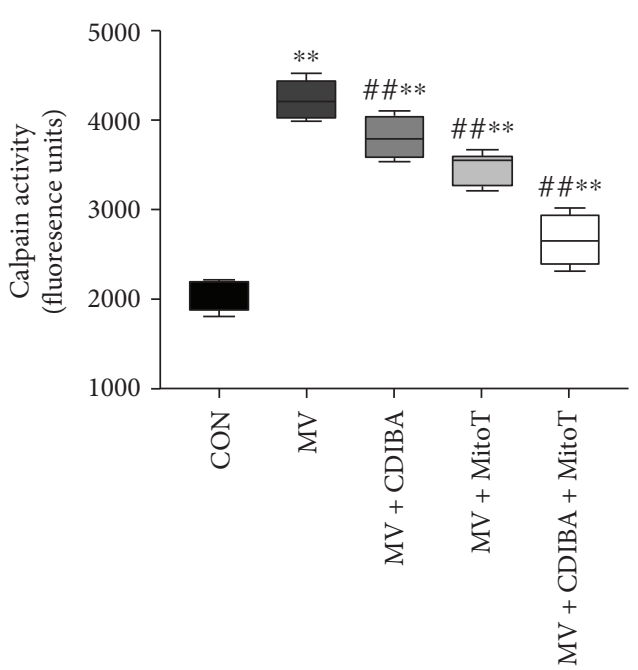

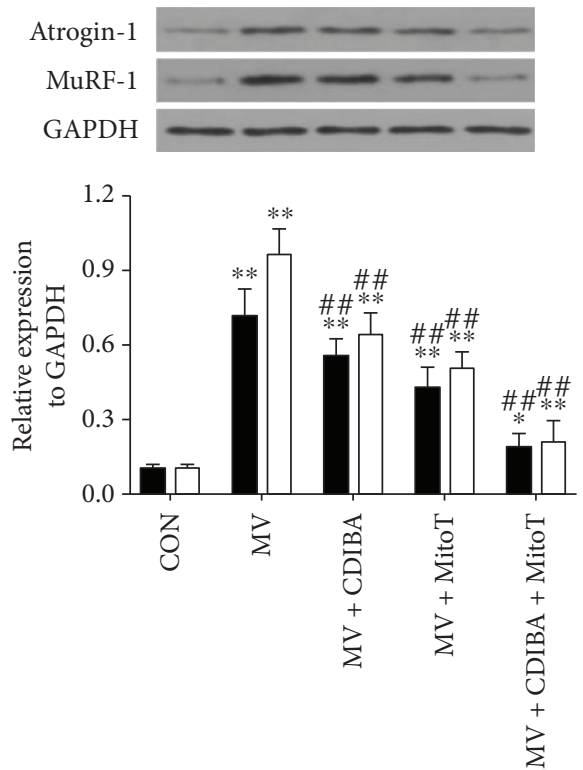

- Atrogin-1

MuRF-1

(b)
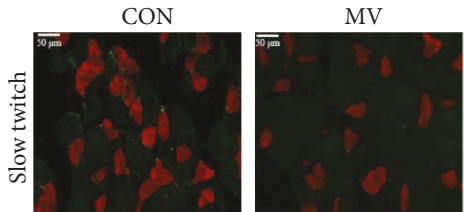

$\mathrm{MV}+\mathrm{CDIBA}$

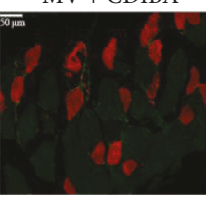

MV + MitoT
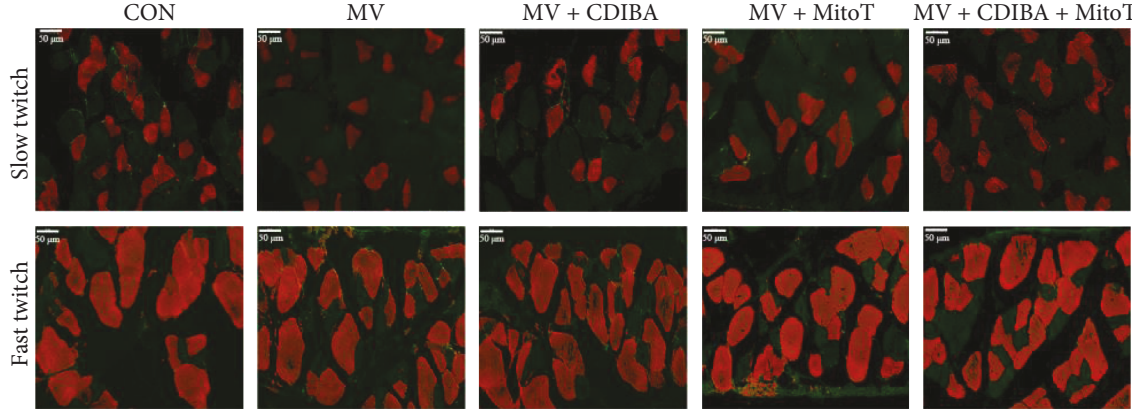

(c)
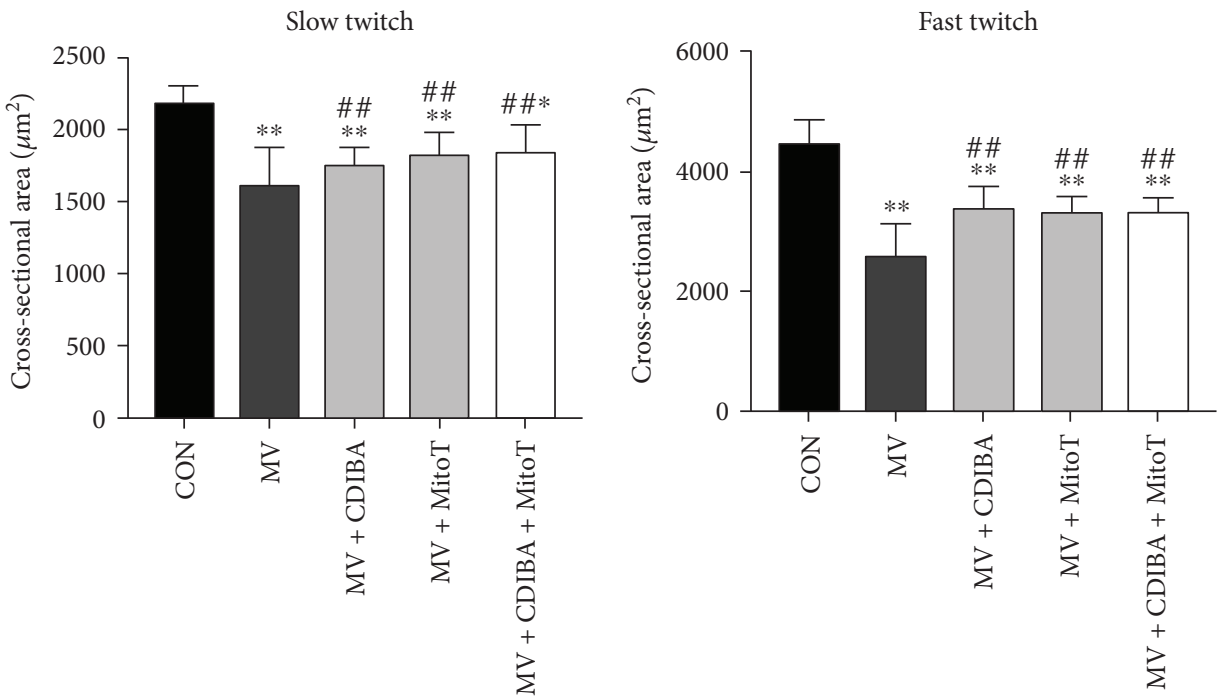

(d)

(e)

FIGURE 2: Protein degradation and atrophy of the diaphragm following MV. (a) calpain activity assay. (b) Western blots for Atrogin-1 and MuRF-1 expressions; (c) immunofluorescence staining for diaphragm muscle fibers; (d) cross-sectional area of slow twitch fibers; (e) cross-sectional area of fast twitch fibers. $\mathrm{MV}=$ mechanical ventilation; cPLA2 = cytosolic phospholipase A2; CDIBA = 4- $\{2$-[5-chloro-1(diphenylmethyl)-2-methyl-1H-indol-3-yl]-ethoxy\} benzoic acid, a specific cPLA2 inhibitor; MitoTEMPO=a mitochondria-targeted antioxidant; ${ }^{*} p<0.05$ and ${ }^{* *} p<0.01$ vs. the control group; ${ }^{\# \#} p<0.01$ vs. the MV group. 


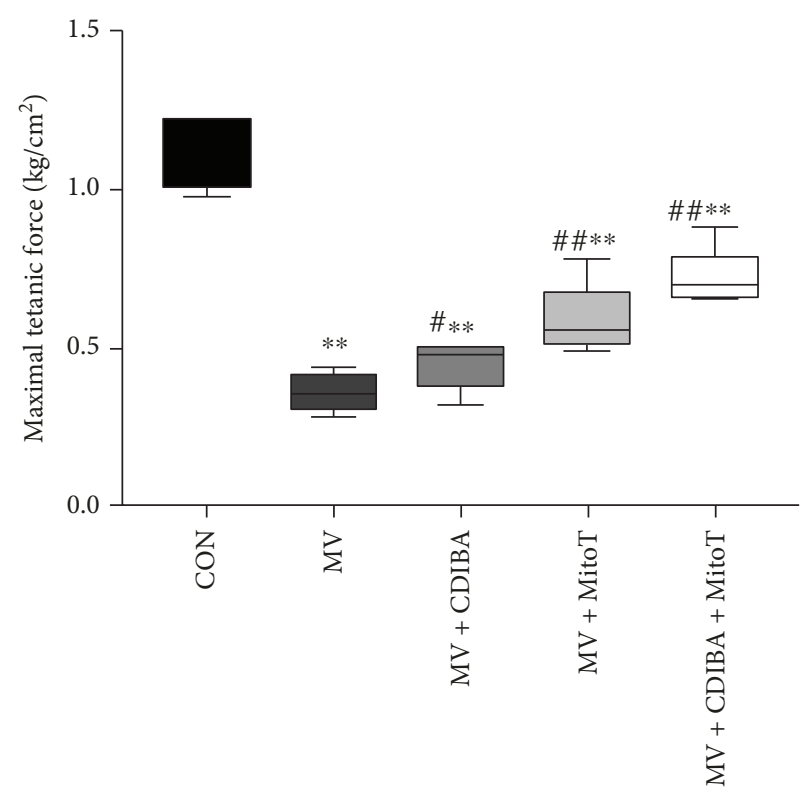

(a)

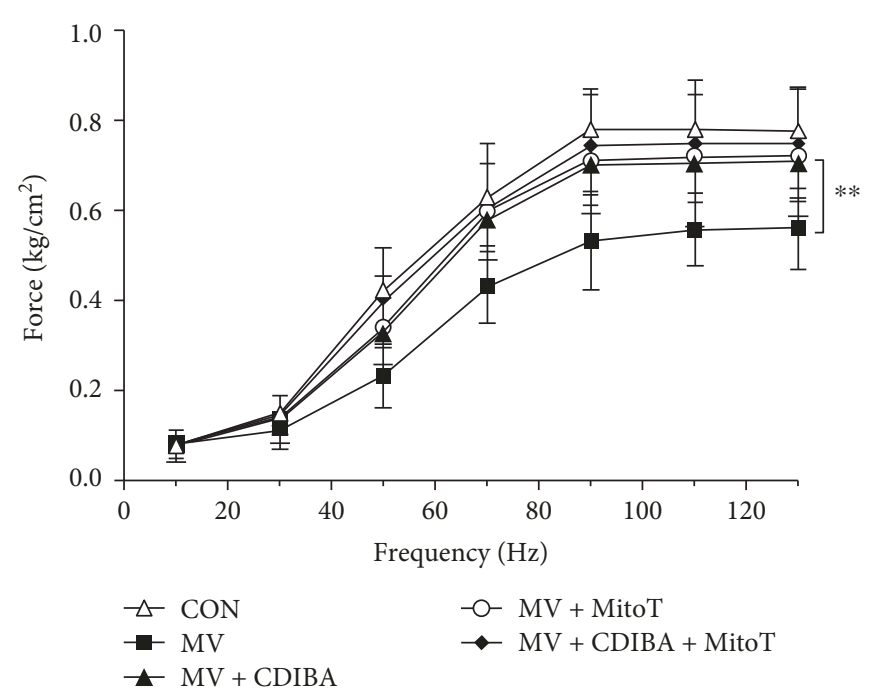

(b)

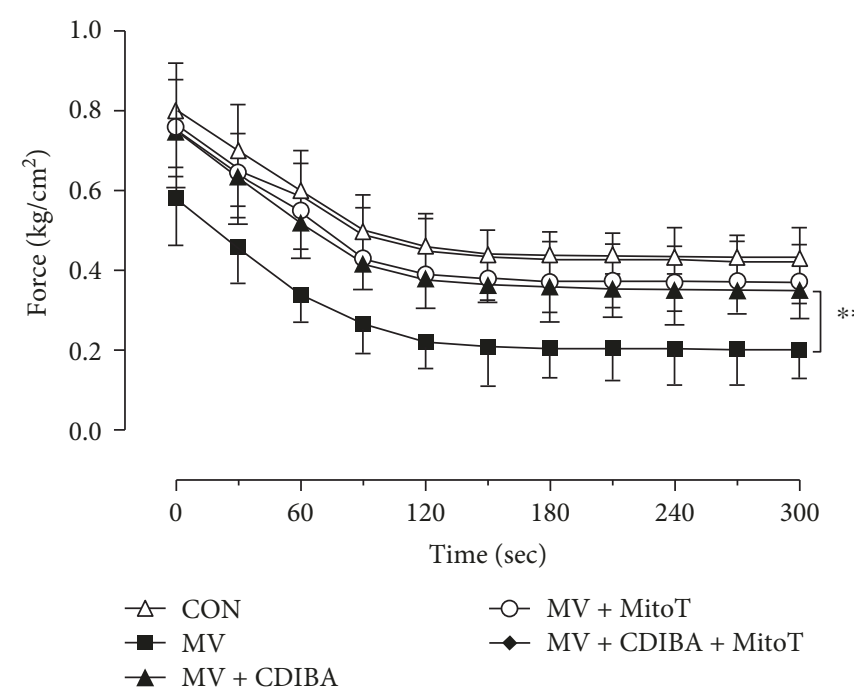

(c)

Figure 3: Diaphragm weakness after prolonged MV. (a) Maximal tetanic forces; (b) force-frequency curve; (c) fatigue tolerance test. $\mathrm{MV}=$ mechanical ventilation; $\mathrm{cPLA} 2=$ cytosolic phospholipase A2; CDIBA $=4-\{2$-[5-chloro-1-(diphenylmethyl) $-2-$ methyl- $1 \mathrm{H}$-indol-3-yl] ethoxy benzoic acid, a specific cPLA2 inhibitor; MitoTEMPO = a mitochondria-targeted antioxidant. ${ }^{* *} p<0.01$ vs. the control group; ${ }^{\# \#} p<0.01$ vs. the MV group.

activation mediated mitochondrial ROS generation and its subsequent events including protein degradation, muscle atrophy, and weakness.

\subsection{Diaphragmatic Hypoxia Contributed to CPLA2 Activation} and Mitochondrial ROS Generation. In this study, we used a laser Doppler machine and probes to determine the microvascular blood flow in the soleus and diaphragm following MV. As seen in Figure 4(a), the perfusion units (PU) were significantly decreased at the end of ventilation compared with the baseline in the MV (768 \pm 26 vs. $600 \pm 54 \mathrm{PU}, p<$ $0.05)$ group. However, there were no significant changes in $\mathrm{PU}$ of the soleus in ventilated animals (Figure 4(b)). In addition, treatments with CDIBA and/or MitoTEMPO did not alter diaphragm tissue perfusion compared with the MV group ( $p>0.05$, respectively) (Figure 4(c)). Next, we checked whether MV induced tissue hypoxia in the diaphragm. Western blot analysis showed that MV for 12 hours induced apparent expression of HIF- $1 \alpha$ in the diaphragm but not in the soleus (Figure 4(d)). These results suggest that prolonged MV induced hypoxia in the diaphragm but not in the soleus. Hypoxia, as a complex stress, is able to induce cellular oxidative stress and cPLA2 activation. Therefore, we performed an in vitro study to investigate the possible association between hypoxia and cPLA2 activation in $\mathrm{C} 2 \mathrm{C} 12$ myoblast cells. As seen in Figures 5(a) and 5(b), hypoxia 


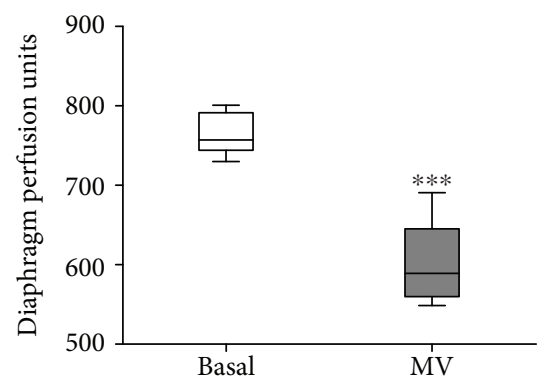

(a)

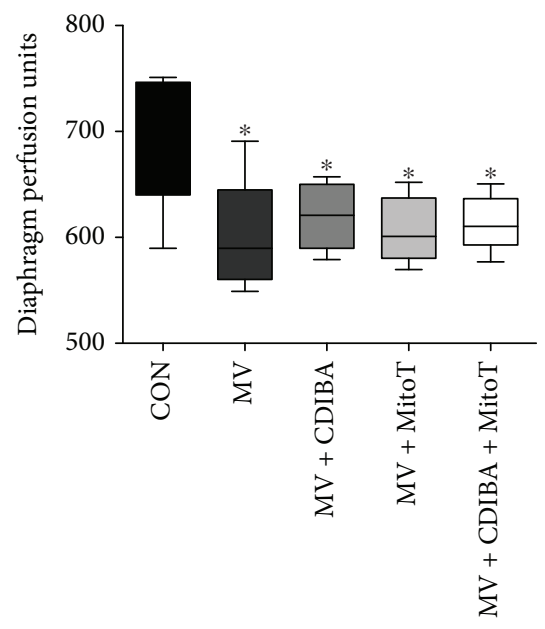

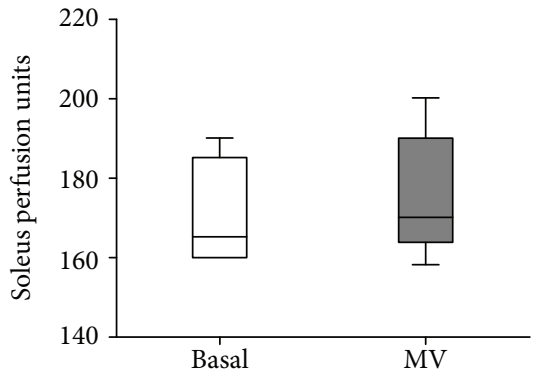

(b)

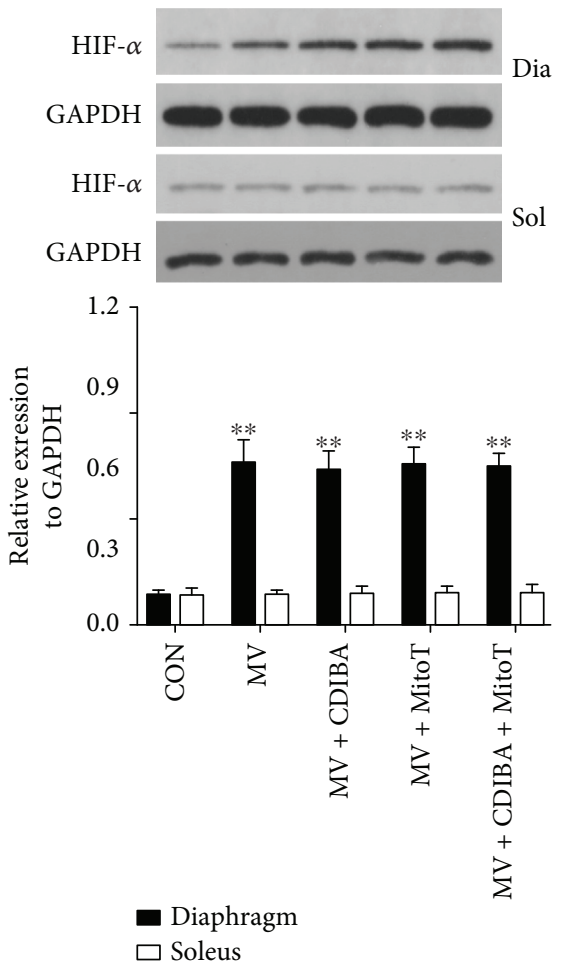

(d)

Figure 4: Ventilator decreased diaphragm perfusion. (a) Laser Doppler for diaphragm perfusion; (b) laser Doppler for soleus perfusion; (c) effects of CDIBA and MitoTEMPO on diaphragm perfusion after 12 hours of MV; (d) western blots for HIF-1 $\alpha$ in the diaphragm and soleus. $\mathrm{MV}=$ mechanical ventilation; cPLA2 = cytosolic phospholipase A2; CDIBA = 4- 2 -[5-chloro-1-(diphenylmethyl)-2-methyl-1H-indol-3-yl]ethoxy benzoic acid, a specific cPLA2 inhibitor; MitoTEMPO $=$ a mitochondria-targeted antioxidant. ${ }^{*} p<0.05$ and ${ }^{* *} p<0.01$ vs. the control group; ${ }^{* * *} p<0.001$ vs. basal.

induced cPLA 2 phosphorylation and increased CPLA2 activities in a time-dependent manner. In addition, administration of either CPLA2 inhibitor AACOCF3 or mitochondriatargeted antioxidant SS31 significantly downregulated hypoxia-induced CPLA2 activation in vitro (Figure $5(\mathrm{c})$ ). Importantly, MitoSOX assays suggested that hypoxia was able to induce mitochondrial ROS generation in vitro, which can be also diminished by the treatment of AACOCF3 or SS31 (Figures 5(d) and 5(e)). Furthermore, hypoxia for 12 hours was able to induce calpain activation in $\mathrm{C} 2 \mathrm{C} 12$ cells, which can also be downregulated by the treatment of AACOCF3 and/or SS31 (Figure 5(f)). Collectively, these results suggest that hypoxia serves as a possible contributor to the cPLA2 activation and mitochondrial ROS generation in the diaphragm after MV. In addition, this in vitro study also confirmed the positive interaction between cPLA2 activation and mitochondrial ROS generation.

\section{Discussion}

The major findings of this study can be summarized as follows: (1) prolonged MV for 12 hours is able to induce cPLA2 activation in the rat diaphragm; (2) ventilator-induced cPLA2 activation positively interacts with mitochondrial ROS generation, which promotes the development of VIDD; and (3) MV decreases diaphragm tissue perfusion and induces expression of HIF- $1 \alpha$, and diaphragm hypoxia possibly contributes to cPLA2 activation and mitochondrial ROS generation following MV.

The imbalance between protein synthesis and proteolysis occurred in the diaphragm following prolonged MV results 

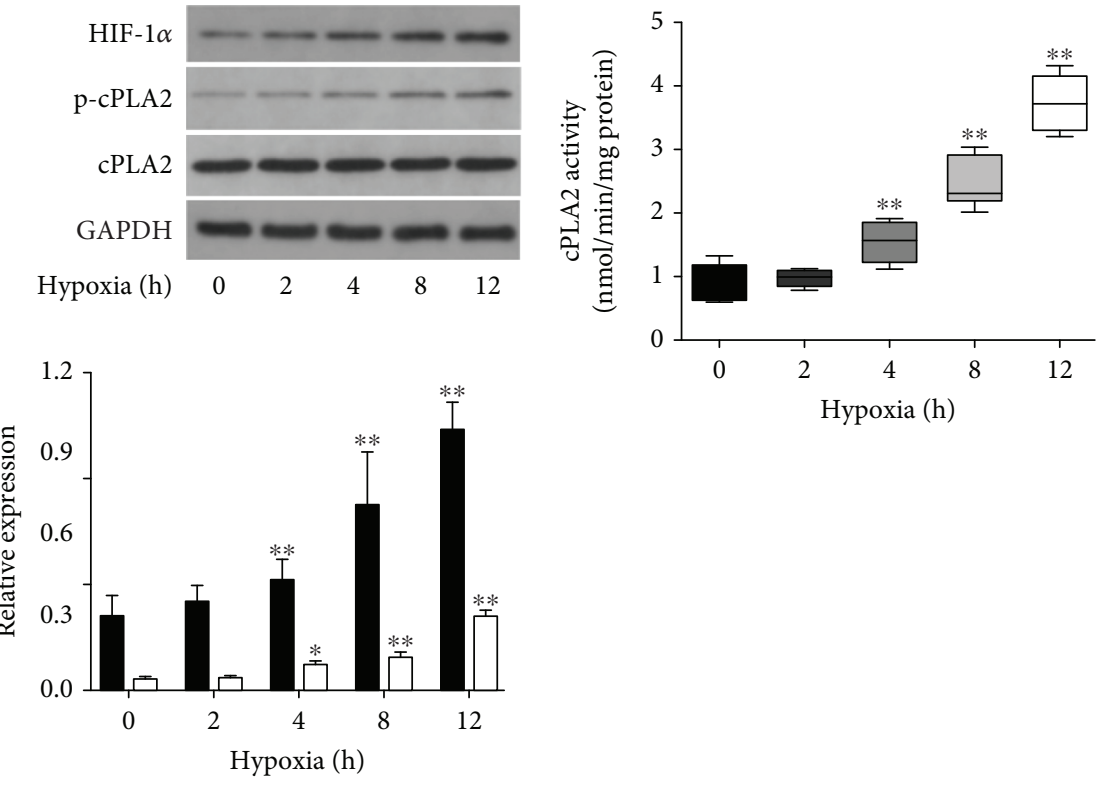

$$
\begin{aligned}
& \square \text { p-cPLA2/cPLA2 } \\
& \square \text { HIF-1 } 1 \alpha / \text { GAPDH }
\end{aligned}
$$

(a)

(b)
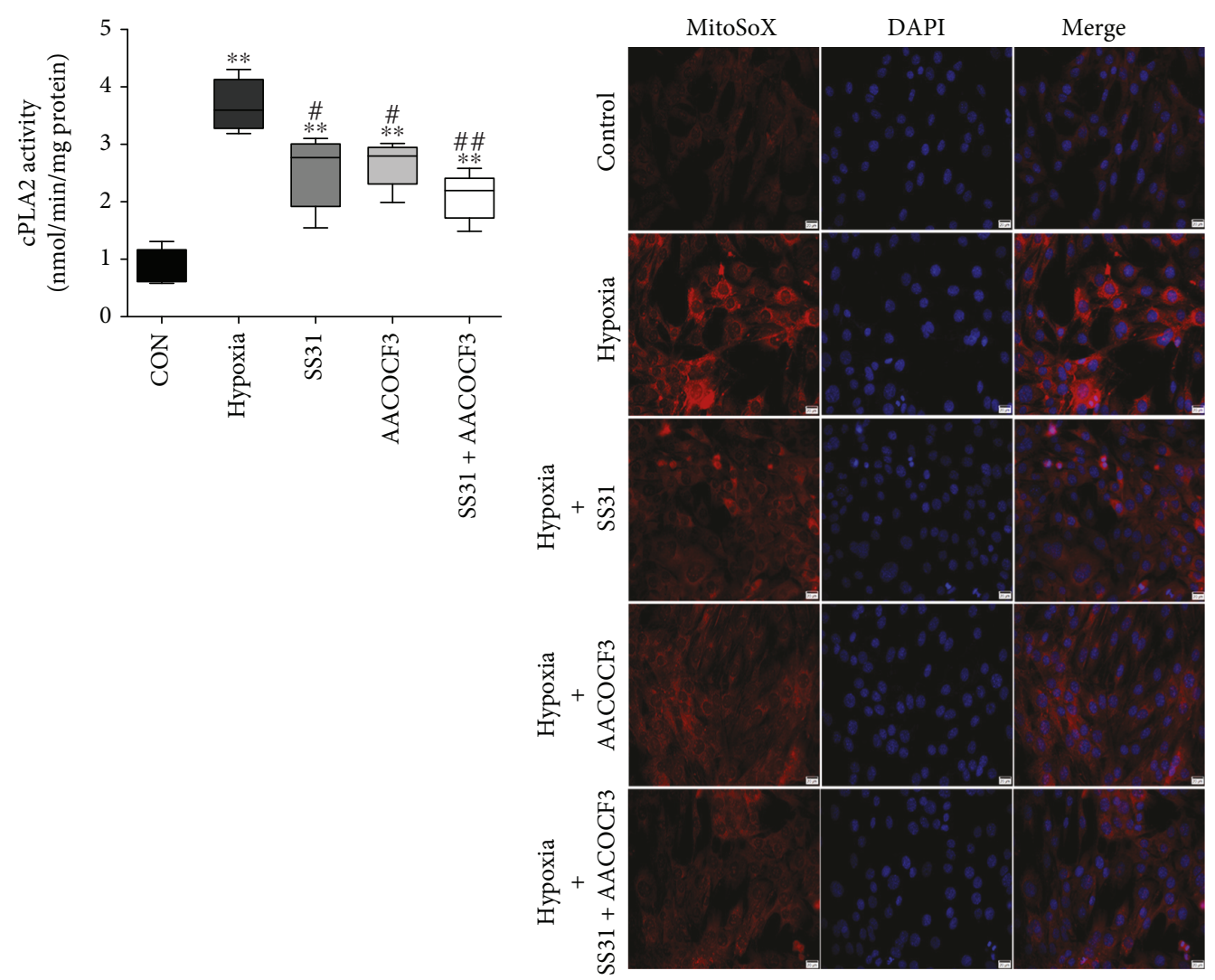

(c)

(d)

FIgure 5: Continued. 


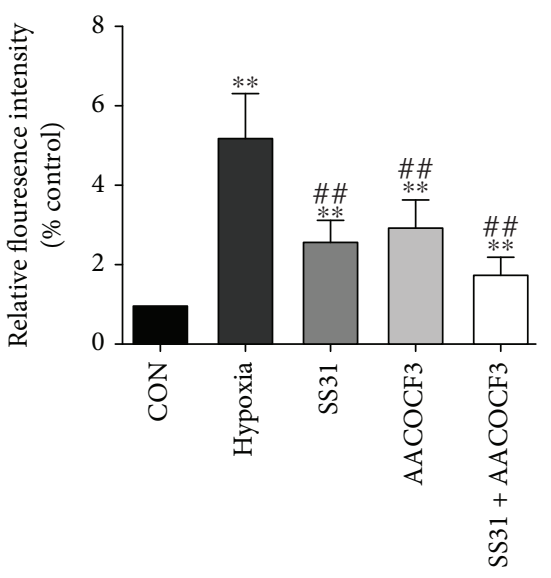

(e)

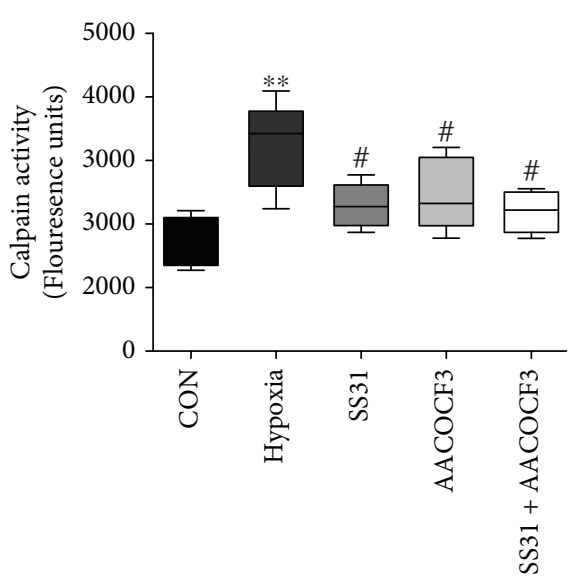

(f)

FIGURE 5: Hypoxia induced cPLA2 activation and mitochondrial ROS generation in vitro. (a) Western blots for HIF- $1 \alpha$ expressions in C2C12 cells; (b) changes of cPLA2 activities in $\mathrm{C} 2 \mathrm{C} 12$ cells underwent indicated time of hypoxia; (c) effects of AACOCF3 and SS31 on cPLA2 activities in $\mathrm{C} 2 \mathrm{C} 12$ cells after 12 hours of hypoxia; (d) MitoSOX assay for the detection of mitochondrial ROS generation in C2C12 cells; (e) fluorescence intensities of MitoSOX; (f) effects of AACOCF3 and SS31 on calpain activities in C2C12 cells after 12 hours of hypoxia. AACOCF, a specific inhibitor of cPLA2; SS31, a mitochondria-targeted antioxidant. ${ }^{*} p<0.05$ and ${ }^{* *} p<0.01$ vs. the control group; ${ }^{\#} p<0.05$ and ${ }^{\# \#} p<0.01$ vs. the MV group.

in muscle atrophy and DD. It has been shown that the upstream regulatory signaling of these proteolytic events is linked to the mitochondrion-derived ROS production in the diaphragm $[7,15]$. In addition, elevated mitochondrial oxidative stress (MOS) has been observed in both animal models and human samples [16]. Importantly, a previous study has demonstrated that MOS is sufficient to trigger the proteolytic process in vitro [15]. In the present study, we observed that prolonged $\mathrm{MV}$ increased mitochondrial $\mathrm{H}_{2} \mathrm{O}_{2}$ production and calpain activation in the diaphragm. The hypothesis that prolonged MV induced cPLA2 activation in the diaphragm has been confirmed in this study. Due to the key role of CPLA2 in mediating lipid mediator production and its widespread tissue expression, it has been implicated in regulating homeostatic processes and disease pathogenesis throughout all organ systems. Several studies have demonstrated the involvement of CPLA2 in diverse pathological processes including skeletal muscle atrophy. cPLA $2 \alpha^{-1-}$ mice are known to have increased striated muscle growth [17]. In addition, cPLA2 mediated fatty acid hydroperoxide generation by mitochondria in denervation-associated muscle atrophy [18]. The relation between the cPLA2 activation and diaphragm muscle atrophy may relate to the mitochondrial oxidative stress. A previous study suggested that the activation of phospholipase A2 is involved in indomethacin-induced damage in Caco-2 cells [19]. In that study, cytosolic phospholipase A2 activation was presented with the increases of oxidative stress in cells treated with indomethacin. Zhu et al. reported that the $\mathrm{A} \beta_{1-42}$-mediated increase in mitochondrial ROS production in astrocytes was suppressed by MAFP, a specific CPLA2 inhibitor [20]. In addition, CPLA2 is one of the constitutively active PLA2s that serve as important mediators for the release of polyunsaturated fatty acids, including arachidonic acid (AA) and docosahexaenoic acid (DHA) from membrane phospholipids $[21,22]$. AA has been shown to induce ROS generation through activation of NADPH oxidases
(NOXs), which may play a key role in the expression of heme oxygenase-1 (HO-1) [23]. In addition, DHA has been reported to trigger ROS generation and finally induce oxidative stressinduced growth inhibitor 1 (OSGIN1) expression in breast cancer cells [24]. Collectively, all these studies suggest that cPLA2 plays an important role in ROS production in different pathological processes. In our study, we observed that the cPLA2 activity was positively associated with the total and mitochondrial ROS production in the diaphragm. This indicated that CPLA2 promoted MV-induced oxidative stress in the diaphragm via inducing mitochondrion-derived ROS production.

Another important finding of this study is the positive feedback loop between cPLA2 activation and mitochondrial ROS production. The mitochondrial ROS generation in the diaphragm was dependent on the activation of cPLA2, whereas treatments with antioxidants also reduced cPLA2 activities. In addition, the cell study showed that exogenous $\mathrm{H}_{2} \mathrm{O}_{2}$ induced cPLA2 activation, and specific inhibition of mitochondrial ROS using SS31 reduced $\mathrm{H}_{2} \mathrm{O}_{2}$-induced activation of CPLA2. Oxidative stress, or ROS, has been reported to promote cPLA2 activation and expression. Cheng and others reported that in human tracheal smooth muscle cells (HTSMCs), cigarette smoke extract (CSE) induced NADPH oxidase activation leading to phosphorylation of p42/p44 MAPK, p38 MAPK, and JNK [25]. These reactions induced NF-kappa B and AP-1 activities, which were essential for CSE-induced cPLA2 gene expression. In addition, oxidative stress or ROS accumulation may increase the permeability of the cell membrane and induce cytosolic calcium accumulation [26]. Moreover, PI3K/MAPK signaling can be activated by oxidative stress under different conditions [27]. Importantly, both cytosolic calcium levels and MAPK phosphorylation are the most important regulators that mediate the activation of cPLA2. This positive feedback between the activation of CPLA2 and mitochondrial 
oxidative stress in the diaphragm during MV might play a very important role in the development of VIDD. Therefore, it is possible that interventions focusing on the blockage of this loop (e.g., a combination of mitochondria-targeted antioxidants and cPLA2 inhibitors) can impact the activation of proteolytic systems and the development of VIDD.

In this study, we first found that ventilator-induced hypoxia serves as a contributor of CPLA2 activation and mitochondrial ROS generation in the diaphragm. In fact, there is a very close association between oxidative stress and hypoxia. Hypoxia is known to elicit excess ROS production and thereby alter redox balance, and both short- and long-term hypoxic exposures have been shown to induce oxidative stress [28, 29]. The combined presence of inactivity and hypoxia is rarely observed in healthy individuals. Here, in addition to the inactivity of the diaphragm during $\mathrm{MV}$, our results showed that prolonged MV decreased blood flow and induced diaphragm tissue hypoxia. Therefore, the combination of inactivity and hypoxia exists in the diaphragm during $\mathrm{MV}$, and those two factors are both able to induce oxidative stress, which is responsible for the activation of proteolytic systems. Our results also showed that diaphragm hypoxia induced cPLA2 activation, which is involved in the development of mitochondrial oxidative stress. However, the mechanism by which hypoxia induced cPLA2 activation remains unclear.

\section{Conclusion}

Collectively, our findings indicated that mitochondrial ROS generation in the diaphragm following prolonged MV was dependent on the activation of cPLA2. On the other side, mitochondrial oxidative stress also promoted diaphragmatic cPLA2 activation. Therefore, the positive feedback loop between CPLA2 and mitochondrial ROS probably accelerates the development of VIDD.

\section{Data Availability}

The data used to support the findings of this study are available from the corresponding author upon request.

\section{Conflicts of Interest}

The authors declare that there are no conflicts of interest.

\section{Authors' Contributions}

Xian-Long Zhou and Xiao-Jun Wei contributed equally to this work.

\section{Acknowledgments}

This study was financially supported by the Emergency Diagnostic \& Therapeutic Center of Central China (Wuhan, Hubei, China).

\section{Supplementary Materials}

sTable 1: body weight, MAP, HR, and RT (mean \pm SD). $\mathrm{BW}=$ body weight;MAP = mean arterial pressure; $\mathrm{HR}=$ heart rate; $\mathrm{RT}=$ rectal temperature; $\mathrm{MV}=$ mechanical ventilation;CDIBA = 4-\{2-[5-chloro-1-(diphenylmethyl)-2-methyl$1 \mathrm{H}$-indol3-yl]-ethoxy $\}$ benzoic acid (cPLA2 inhibitor): MitoT = MitoTEMPO. BW was determined before the experiment. Values of MAP, HR, and RT at the end of study are presented here. (Supplementary Materials)

\section{References}

[1] C. S. H. Sassoon, V. J. Caiozzo, A. Manka, and G. C. Sieck, "Altered diaphragm contractile properties with controlled mechanical ventilation," Journal of Applied Physiology, vol. 92, no. 6, pp. 2585-2595, 2002.

[2] S. Jaber, M. Sebbane, C. Koechlin et al., "Effects of short vs. prolonged mechanical ventilation on antioxidant systems in piglet diaphragm," Intensive Care Medicine, vol. 31, no. 10, pp. 1427-1433, 2005.

[3] S. K. Powers, R. A. Shanely, J. S. Coombes et al., "Mechanical ventilation results in progressive contractile dysfunction in the diaphragm," Journal of Applied Physiology, vol. 92, no. 5, pp. 1851-1858, 2002.

[4] J. M. McClung, A. N. Kavazis, K. C. DeRuisseau et al., "Caspase-3 regulation of diaphragm myonuclear domain during mechanical ventilation-induced atrophy," American Journal of Respiratory and Critical Care Medicine, vol. 175, no. 2, pp. 150-159, 2007.

[5] H. Tang and J. B. Shrager, "The signaling network resulting in ventilator-induced diaphragm dysfunction," American Journal of Respiratory Cell and Molecular Biology, vol. 59, no. 4, pp. 417-427, 2018.

[6] M. A. Whidden, A. J. Smuder, M. Wu, M. B. Hudson, W. B. Nelson, and S. K. Powers, "Oxidative stress is required for mechanical ventilation-induced protease activation in the diaphragm," Journal of Applied Physiology, vol. 108, no. 5, pp. 1376-1382, 2010.

[7] A. N. Kavazis, E. E. Talbert, A. J. Smuder, M. B. Hudson, W. B. Nelson, and S. K. Powers, "Mechanical ventilation induces diaphragmatic mitochondrial dysfunction and increased oxidant production," Free Radical Biology \& Medicine, vol. 46, no. 6, pp. 842-850, 2009.

[8] D. Nethery, L. A. Callahan, D. Stofan, R. Mattera, A. DiMarco, and G. Supinski, "PLA 2 dependence of diaphragm mitochondrial formation of reactive oxygen species," Journal of Applied Physiology, vol. 89, no. 1, pp. 72-80, 2000.

[9] G. S. Supinski, A. P. Alimov, L. Wang, X. H. Song, and L. A. Callahan, "Calcium-dependent phospholipase $\mathrm{A}_{2}$ modulates infection-induced diaphragm dysfunction," American Journal of Physiology-Lung Cellular and Molecular Physiology, vol. 310, no. 10, pp. L975-L984, 2016.

[10] R. T. Davis, C. S. Bruells, J. N. Stabley, D. J. McCullough, S. K. Powers, and B. J. Behnke, "Mechanical ventilation reduces rat diaphragm blood flow and impairs oxygen delivery and uptake," Critical Care Medicine, vol. 40, no. 10, pp. 28582866, 2012.

[11] D. Wu and P. Yotnda, "Induction and testing of hypoxia in cell culture," Journal of Visualized Experiments, no. 54, article e2899, 2011. 
[12] M. L. Garcia-Cazarin, N. N. Snider, and F. H. Andrade, "Mitochondrial isolation from skeletal muscle," Journal of Visualized Experiments, no. 49, article e2452, 2011.

[13] S. Levine, T. Nguyen, N. Taylor et al., "Rapid disuse atrophy of diaphragm fibers in mechanically ventilated humans," The New England Journal of Medicine, vol. 358, no. 13, pp. 1327$1335,2008$.

[14] M. Le Dinh, S. Carreira, J. Obert et al., "Prolonged mechanical ventilation worsens sepsis-induced diaphragmatic dysfunction in the rat," PLoS One, vol. 13, no. 8, article e0200429, 2018.

[15] H. Tang, M. Lee, M. T. Budak et al., "Intrinsic apoptosis in mechanically ventilated human diaphragm: linkage to a novel Fos/FoxO1/Stat3-Bim axis," The FASEB Journal, vol. 25, no. 9, pp. 2921-2936, 2011.

[16] S. K. Powers, A. N. Kavazis, and S. Levine, "Prolonged mechanical ventilation alters diaphragmatic structure and function," Critical Care Medicine, vol. 37, pp. S347-S353, 2009.

[17] S. Haq, H. Kilter, A. Michael et al., "Deletion of cytosolic phospholipase $\mathrm{A}_{2}$ promotes striated muscle growth," Nature Medicine, vol. 9, no. 7, pp. 944-951, 2003.

[18] A. Bhattacharya, F. L. Muller, Y. Liu et al., "Denervation induces cytosolic phospholipase $\mathrm{A}_{2}$-mediated fatty acid hydroperoxide generation by muscle mitochondria," The Journal of Biological Chemistry, vol. 284, no. 1, pp. 46-55, 2009.

[19] N. Sivalingam, J. Basivireddy, A. B. Pulimood, K. A. Balasubramanian, and M. Jacob, "Activation of phospholipase $A_{2}$ is involved in indomethacin-induced damage in Caco-2 cells," Toxicology In Vitro, vol. 23, no. 5, pp. 887-896, 2009.

[20] D. Zhu, Y. Lai, P. B. Shelat, C. Hu, G. Y. Sun, and J. C. M. Lee, "Phospholipases $\mathrm{A}_{2}$ mediate amyloid- $\beta$ peptide-induced mitochondrial dysfunction," The Journal of Neuroscience, vol. 26, no. 43, pp. 11111-11119, 2006.

[21] C. C. Leslie, "Cytosolic phospholipase $\mathrm{A}_{2}$ : physiological function and role in disease," Journal of Lipid Research, vol. 56, no. 8, pp. 1386-1402, 2015.

[22] J. E. Burke and E. A. Dennis, "Cytosolic phospholipase $\mathrm{A}_{2}$ : physiological function and role in disease," Journal of Lipid Research, vol. 50, Supplement, pp. S237-S242, 2009.

[23] C. C. Lin, C. C. Yang, Y. W. Chen, L. D. Hsiao, and C. M. Yang, "Arachidonic acid induces ARE/Nrf2-dependent heme oxygenase-1 transcription in rat brain astrocytes," Molecular Neurobiology, vol. 55, no. 4, pp. 3328-3343, 2018.

[24] C. H. Tsai, Y. C. Shen, H. W. Chen et al., "Docosahexaenoic acid increases the expression of oxidative stress-induced growth inhibitor 1 through the PI3K/Akt/Nrf2 signaling pathway in breast cancer cells," Food and Chemical Toxicology, vol. 108, pp. 276-288, 2017.

[25] S. E. Cheng, S. F. Luo, M. J. Jou et al., "Cigarette smoke extract induces cytosolic phospholipase $\mathrm{A}_{2}$ expression via $\mathrm{NADPH}$ oxidase, MAPKs, AP-1, and NF- $\kappa$ B in human tracheal smooth muscle cells," Free Radical Biology and Medicine, vol. 46, no. 7, pp. 948-960, 2009.

[26] A. Görlach, K. Bertram, S. Hudecova, and O. Krizanova, "Calcium and ROS: a mutual interplay," Redox Biology, vol. 6, pp. 260-271, 2015.

[27] J. Yu, F. Liu, P. Yin et al., "Involvement of oxidative stress and mitogen-activated protein kinase signaling pathways in heat stress-induced injury in the rat small intestine," Stress, vol. 16, no. 1, pp. 99-113, 2013.
[28] P. Joanny, J. Steinberg, P. Robach et al., "Operation Everest III (Comex'97): the effect of simulated sever hypobaric hypoxia on lipid peroxidation and antioxidant defence systems in human blood at rest and after maximal exercise," Resuscitation, vol. 49, no. 3, pp. 307-314, 2001.

[29] A. Dosek, H. Ohno, Z. Acs, A. W. Taylor, and Z. Radak, "High altitude and oxidative stress," Respiratory Physiology \& Neurobiology, vol. 158, no. 2-3, pp. 128-131, 2007. 


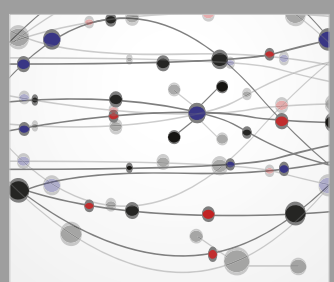

The Scientific World Journal
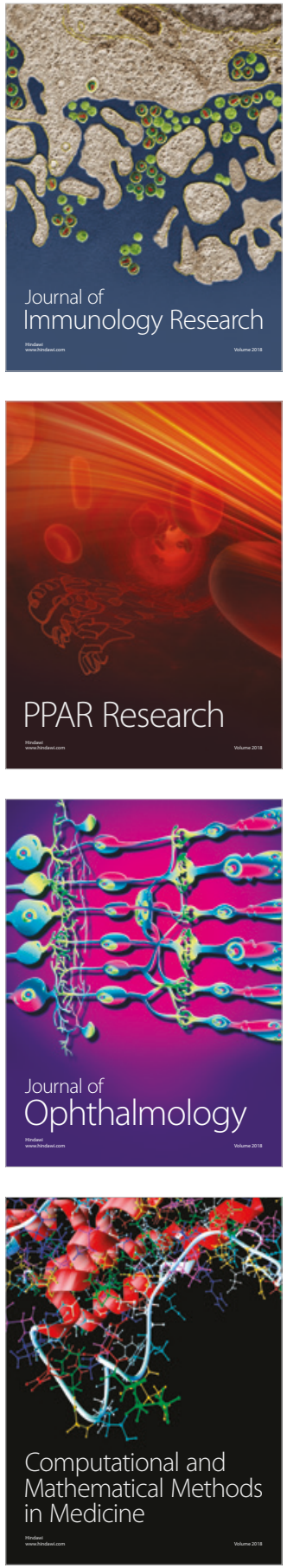

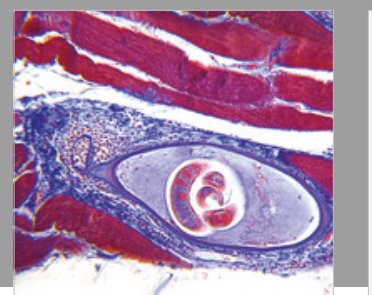

Gastroenterology Research and Practice

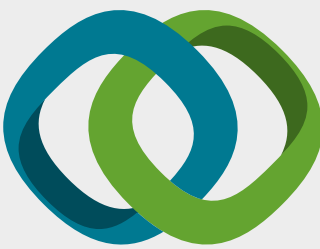

\section{Hindawi}

Submit your manuscripts at

www.hindawi.com
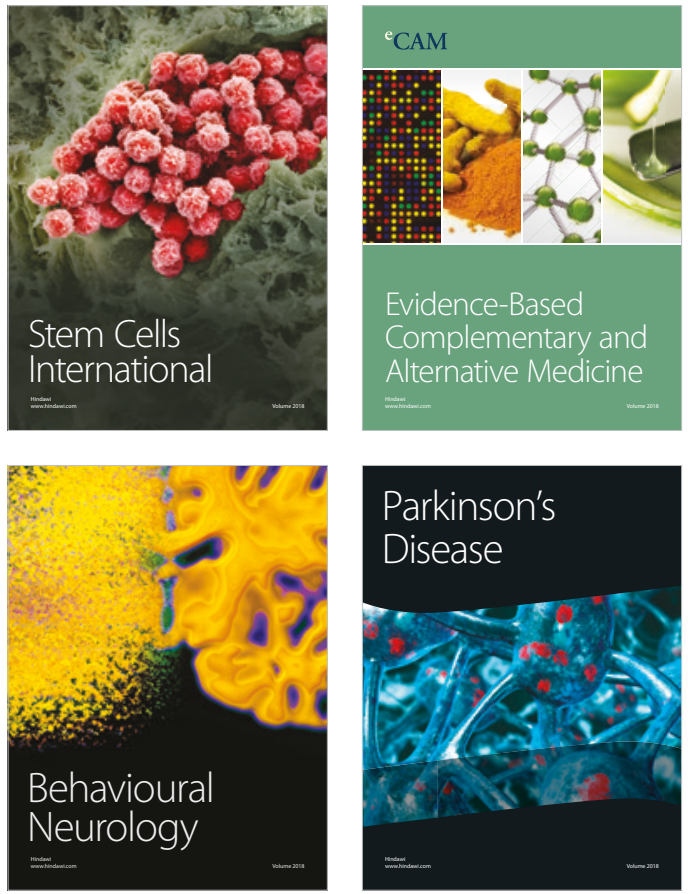

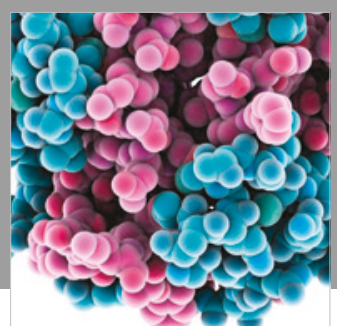

ournal of

Diabetes Research

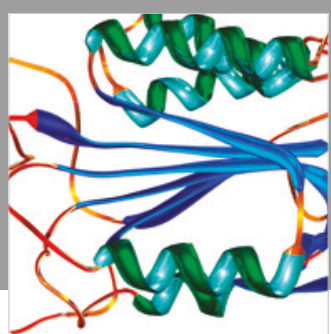

Disease Markers
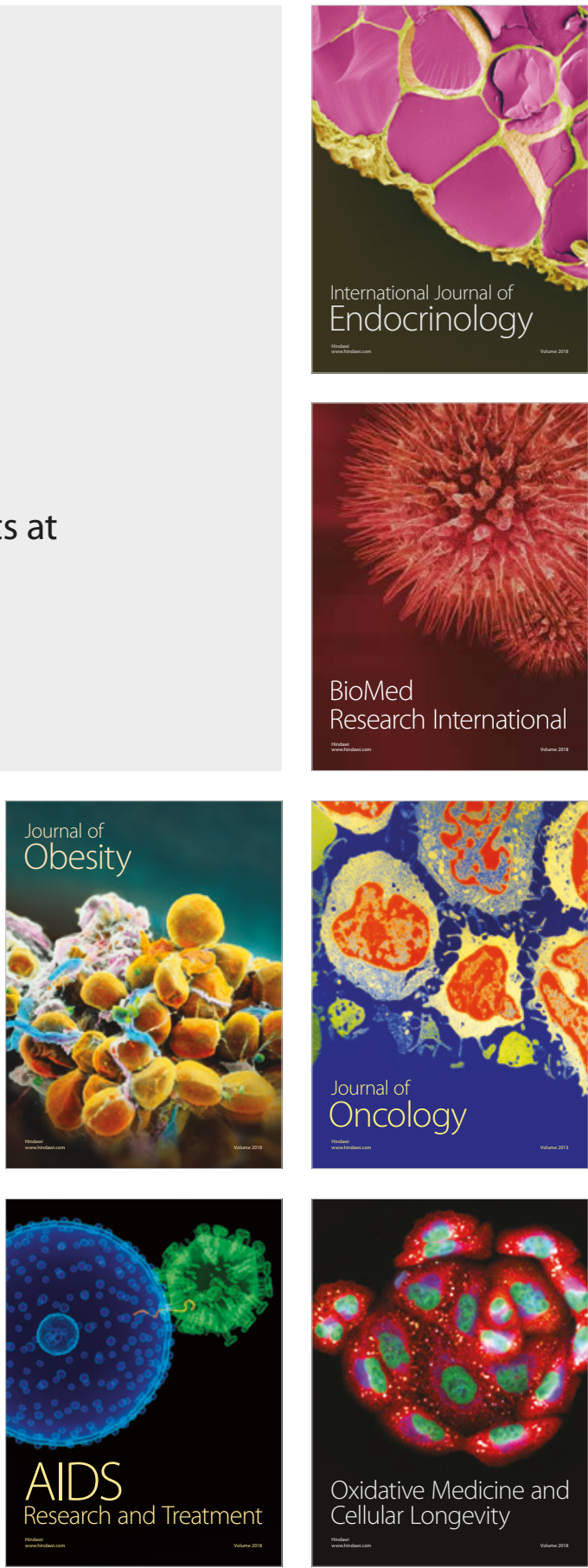